\title{
Production of high energy-solid density electron bunches and hard $\gamma$-quanta and positron fluxes by ultrarelativistic lasers in solid-plasma targets
}

\author{
H. K. Avetissian, ${ }^{1}$ H. H. Matevosyan, ${ }^{2}$ G. F. Mkrtchian, ${ }^{1}$ and Kh. V. Sedrakian ${ }^{1}$ \\ ${ }^{1}$ Centre of Strong Fields Physics, Yerevan State University, 1 A. Manukian, Yerevan 0025, Armenia \\ ${ }^{2}$ Plasma Theory Group, Institute of Radiophysics and Electronics, 0203 Ashtarak, Armenia
}

(Received 27 July 2015; published 11 December 2015)

\begin{abstract}
We consider nonlinear interaction of superpower laser pulses of relativistic intensities with nanolayers and solid-plasma targets toward the production of high energy-density electron and positron bunches along with high energy photon fluxes. It is shown that petawatt lasers are capable of producing via two-target scheme high density field-free electron/positron bunches and substantial amounts of $\gamma$-quanta with energies up to $200 \mathrm{MeV}$. For actual supershort and tightly focused-strongly nonplane ultrarelativistic laser pulses of linear and circular polarizations, the $3 \mathrm{D} 3 \mathrm{~V}$ problem is solved via numerical simulations.
\end{abstract}

DOI: 10.1103/PhysRevSTAB.18.121301

PACS numbers: 52.38.Ph, 41.75.Jv, 41.75.Ht

\section{INTRODUCTION}

Acceleration of electrons with superpower laser beams (at present-of relativistic intensities) and their interaction with the matter in ultrashort space-time scales have attracted broad interest over the last two decades, stimulated by the continued progress made in laser technology. In many laboratories [1-4] compact lasers can deliver 1-10 petawatt short pulses, with focused intensities as high as $10^{22} \mathrm{~W} \mathrm{~cm}^{-2}$. Next generation multipetawatt and exawatt optical laser systems [5-7] will be capable to deliver ultrahigh intensities exceeding $10^{25} \mathrm{~W} \mathrm{~cm}^{-2}$, which are well above the ultrarelativistic regime of electron-laser interaction. Laser intensities at which an electron becomes relativistic is defined by the condition $\xi \gtrsim 1$, where $\xi=$ $e E \chi / m c^{2}$ is the relativistic invariant dimensionless parameter of a wave-particle interaction ( $e$ is the elementary charge, $c$ is the light speed in vacuum, and $m$ is the electron mass) and represents the work of the wave electric field with strength $E$ on a wavelength $\lambda(\lambda=\lambda / 2 \pi)$ in the units of electron rest energy. Laser intensities expressed via $\xi$ can be estimated as:

$$
I_{r}=\xi^{2} \times 1.37 \times 10^{18} \mathrm{~W} \mathrm{~cm}^{-2}[\lambda / \mu \mathrm{m}]^{-2} .
$$

Thus, available optical lasers provide $\xi$ up to 100 , meanwhile with the next generation of laser systems we will be able to exploit beams at $\xi>1000$. In such ultrastrong laser fields, electrons can reach ultrarelativistic energies. However to obtain ultrarelativistic net energies for fieldfree electrons one should bypass the limitations imposed by the well known Lawson-Woodward theorem [8]. One way

Published by the American Physical Society under the terms of the Creative Commons Attribution 3.0 License. Further distribution of this work must maintain attribution to the author $(s)$ and the published article's title, journal citation, and DOI. to get net energy exchange is to use coherent processes of laser-particle interaction with the additional resonances. Among those the induced Cherenkov, Compton, and undulator processes are especially of interest $[9,10]$. In these induced processes one can obtain moderate relativistic electron beams of low energy spreads and emittances due to the threshold character of nonlinear resonance in the strong wave field. Besides, the use of a plasma medium [11] is a promising way of achieving laser-driven electron acceleration. However, laser-plasma accelerator schemes face problems connected with the inherent instabilities in laser-plasma interaction processes. The spectrum of direct acceleration mechanisms of charged particles by a single laser pulse is very restricted, since one should use the laser beams focused to subwavelength waist radii, or use subcycle laser pulses, or use radially polarized lasers. All these scenarios with different field configurations have been investigated both theoretically and experimentally [12-28]. The Lawson-Woodward theorem can also be bypassed by terminating the field, either by reflection, absorption, or diffraction [29]. The proof of principle experiment of this type has been reported in Ref. [30]. Here, initially relativistic electron beam and a moderately strong laser pulse have been used. To obtain dense enough electron bunches it is reasonable to consider electrons acceleration from nanoscale-solid plasma-targets [31-35] at intensities high enough to separate all electrons from ions [36]. Thus, combining these two schemes one can obtain ultrarelativistic solid density electron bunches. Such bunches can be used to obtain high-flux of positrons, $\gamma$ quanta with possible applications in material science, medicine, and nuclear physics.

In the present work we propose an efficient mechanism for creation of ultrarelativistic field-free electron bunches, $\gamma$-ray and positron beam of high fluxes by a single laser pulse of ultrarelativistic intensities. The scheme is as follows: a laser beam of ultrarelativistic intensities is 
focused onto nanoscale-solid plasma-target with relatively low $\mathcal{Z}$. From this target under the action of ultrashort laser pulse a superdense electron bunch is formed and accelerated up to ultrarelativistic energies. Then we place a high$\mathcal{Z}$ target (tungsten or gold) at the distances where the electron bunch gains maximum energy from the laser pulse. The purpose of the second target is twofold. First, it acts as a reflector for laser pulse, being practically transparent for electron bunch. As a consequence we get field-free ultrarelativistic electron bunch. Second, within this target with sufficiently large thickness the generation of hard $\gamma$-quanta and positron fluxes takes place due to the electron-ion collisions.

The organization of the paper is as follows. In Sec. II for supershort strongly nonplane ultrarelativistic laser pulses of linear and circular polarizations problem is solved via numerical simulations. In Sec. III we present numerical calculations for produced $\gamma$-quanta and positron fluxes. Finally, conclusions are given in Sec. IV.

\section{GENERATION OF ULTRARELATIVISTIC ELECTRON BUNCHES FROM NANOTARGETS}

Here we report on the results of the 3D3V simulations of superintense laser beam interaction with solid-plasmatargets. The illustration of proposed scheme is as follows: a laser beam of ultrarelativistic intensities $(\xi \gg 1)$ is focused onto solid-plasma-target of nanothickness with relatively low $\mathcal{Z}$. For concreteness we take carbon target $(\mathcal{Z}=6)$. Target is assumed to be fully ionized. This is justified since the intensity for full ionization of carbon is about $10^{19} \mathrm{~W} / \mathrm{cm}^{2}$, while we use in simulations intensities at least on two order of magnitude larger. So, the target will become fully ionized before the arrival of the pulse peak. Electrons are assumed to be cold in the target, $T_{e}=0$. The carbon target size is $50 \lambda \times 50 \lambda$ in the $x y$ plane and $d=0.002 \lambda$ in $z$ direction (wave propagation direction). The foil is chosen to be thin enough for the laser beam to push out all electrons from the target. In this case, only electrons are accelerated, while the ions are left unmoved. For this regime [36], the relativistic invariant parameter of the laser field $\xi$ must be larger than the normalized field arising from electron-ion separation: $\eta=\pi\left(n_{e} / n_{c}\right) d / \lambda$. Here $n_{e}$ is the electron density $\left(n_{e} \simeq 6.5 \times 10^{23} \mathrm{~cm}^{-3}\right.$ for carbon) and $n_{c}=m \omega^{2} /\left(4 \pi e^{2}\right)$ is the critical density. We assume laser radiation of $\lambda=800 \mathrm{~nm}$ and therefore $n_{c} \simeq 1.74 \times 10^{21} \mathrm{~cm}^{-3}$. For the chosen thickness $d=1.6 \mathrm{~nm}$ we have $\eta \simeq 2.35$. Thus, to fulfill the condition $\xi \gg \eta$ for all calculations we take $\xi \geq 20$. Although the electron density is overcritical, the target is much thinner than the skin depth and is therefore transparent for the laser beam. Note that the considered scheme requires nonadiabatic laser pulses with a very high intensity contrast ratio $\sim 10^{-12}$ [35], because the prepulses of long duration can destroy the target before the main pulse arrives.

Since 3D3V particle-in-cell (PIC) simulations require supercomputers and are time consuming, we will consider the interaction scheme and range of laser/target parameters when electrons motion can be satisfactorily described in a single-particle picture. To reach this regime one should minimize the other physical effects that may play a negative role in laser accelerated solid-density electron bunches, such as the Coulomb forces (space charge effect), laser field depletion, and radiation reaction/friction. To reduce the Coulomb expansion of the electron sheet, we suppose a relatively small $\eta$, which is proportional to the surface density of electrons $n_{e} d$. Then as was shown in Refs. [36,37], in spite of Coulomb forces, all of the electrons in the laser spot can be synchronously accelerated to ultrarelativistic energies by the first half-cycle of the field. For the distances $\sim 4 \lambda$ electron sheet reaches to Lorentz factors $\gamma=1+\xi^{2} / 2\left(1+\eta^{2}\right)$ [37], which for $\xi=30, \eta=2.35$ gives $\gamma \simeq 70$. The space-charge effects in a bunch are characterized by the beam generalized perveance $\sim 1 / \gamma^{3}$ [38] that is significantly reduced; thus preventing Coulomb explosion of the bunch at the further evolution in the laser field. Although for the acceleration of electrons from solid targets one needs $\xi \gtrsim 3 \eta$, we have taken sufficiently high $\xi$, correspondingly large energy per pulse: $>2.5 \times 10^{14} \mathrm{MeV}$, to avoid the laser pulse depletion effects. Assuming that all electrons on the laser spot are accelerated to $100 \mathrm{MeV}$ energies, it is easy to estimate that the laser energy depletion is $\lesssim 10 \%$. At the interaction of laser beams of relativistic intensities with the electrons, radiation reaction/friction effects may become important. This corresponds to dimensionless laser field amplitude parameter $\xi$ above the values $\xi_{\text {rad }} \simeq \sqrt{3 \lambda /\left(8 \pi r_{e} \gamma\right)}$ [39] $\left(r_{e}\right.$ is the electron classical radius), which for our scheme with $\gamma \sim 200$ corresponds to $\xi_{\text {rad }} \simeq 500$. The latter is considerably larger than the assumed values for $\xi$.

Since in the initial stage of the laser-target interaction process plasma effects are essential, we have verified the process of electron layer blown out from a carbon target by PIC simulations. For this goal, we use the code XOOPIC, which is a relativistic $2 \mathrm{D} 3 \mathrm{~V}$ code based on the PIC method [40]. The simulation box size is $40 \lambda \times 2 \lambda$ in the $y z$ plane. The number of cells is $4000 \times 500$. The total number of macroparticles is about $10^{5}$. The linearly $y$-polarized laser pulse has Gaussian temporal and transverse profiles: $\exp \left(-\varphi^{2} / \delta^{2}-\rho^{2} / w_{0}^{2}\right) ; \varphi=\omega t-k z$, with the pulse width $\delta=7$ and the waist $w_{0}=10 \lambda$. The laser beam is introduced at the left boundary $(-151 \lambda)$, running along the $\mathrm{z}$ axis and focused at the target layer. In Fig. 1, the electron layer blown out from a carbon target is shown at the instant $t=4 \mathrm{fs}$, for $\xi=30$ and $\xi=60$ calculated with 2D PIC code. For comparison we also show results in a single particle approximation when plasma effects are neglected (see below). As is seen from this figure, all electrons are removed from the central part of the nanotarget and are accelerated to relativistic energies. The two results are almost overlapped despite small broadening of the electron layer due to the Coulomb attraction of ions at the initial 

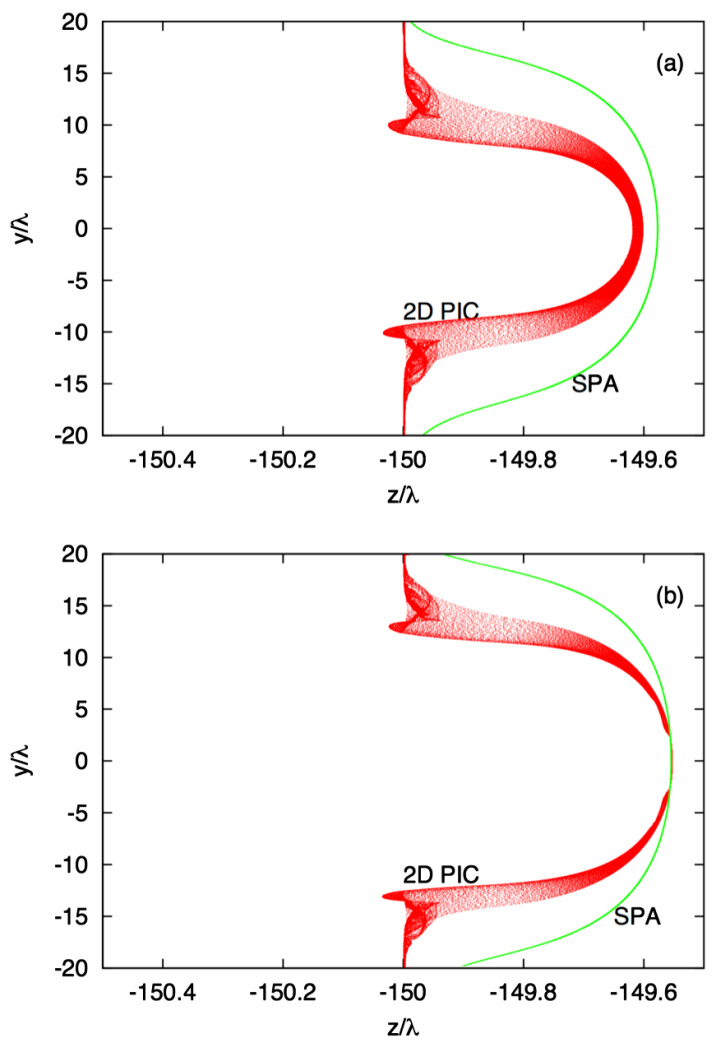

FIG. 1. Electron layer blown out from a nano-film by superstrong laser beam of linear polarization. (a) The $(x-y)$ phase space for electrons at instant 4 fs for $w_{0}=10 \lambda$ and $\xi=30$, calculated with 2D PIC and in the single particle approach (SPA). (b) Same as (a) but for $w_{0}=10 \lambda$ and $\xi=60$.

stage of acceleration. These effects are essential for relatively small laser intensities, meanwhile for stronger laser pulses Fig. 1(b) due to a snowplow action of the laser pulse $[37,41]$ all electrons are compressed. Thus, a single particle approach is somewhat justified, i.e. one can solve relativistic equations of motion for macroparticles

$$
\frac{d \Pi}{d t}=\frac{e}{m c}\left(\mathbf{E}+\frac{\Pi \times \mathbf{H}}{\gamma}\right), \quad \frac{d \gamma}{d t}=\frac{e}{m c} \frac{\Pi \cdot \mathbf{E}}{\gamma}
$$

in the given electric $\mathbf{E}$ and magnetic $\mathbf{H}$ fields. Here we have introduced normalized momentum $\Pi=\mathbf{p} /(m c)$, energy $\gamma=\sqrt{1+\Pi^{2}}$ (Lorentz factor) and have taken into account that charge to mass ratio of macroparticles is the same as for the electron.

The laser beam is focused at the $z=0$ and propagates along the $O Z$. As is clear from the relativistic dynamics Eq. (1), the snowplow action of the laser pulse is conditioned by the ponderomotive force $\sim \Pi \times \mathbf{H}$ which strongly depends on the wave polarization. We will consider as circular, as well as linear polarization of drive laser beam. For circularly polarized lasers, the ponderomotive force is steady and it is expected that dense electron bunch will be stable for a longer time duration than in the case of linear polarization where oscillating part of the ponderomotive force causes strong electron heating [42]. For analytic description of such pulses of linear/circular polarization we will approximate corresponding electromagnetic fields as follow [43]:

$$
\begin{gathered}
E_{x}=\tilde{E}_{0}(\mathbf{r}, t)\left(\cos \varphi_{-}-\frac{z}{z_{R}} \sin \varphi_{-}\right), \\
E_{y}=g \tilde{E}_{0}(\mathbf{r}, t)\left(\sin \varphi_{-}+\frac{z}{z_{R}} \cos \varphi_{-}\right), \\
E_{z}=\frac{\tilde{E}_{0}(\mathbf{r}, t) \lambda}{\pi w^{2}(z)}\left\{\left[g y\left(1-\frac{z^{2}}{z_{R}^{2}}\right)-2 \frac{z x}{z_{R}}\right] \cos \varphi_{-} .\right. \\
\left.+\left[x\left(1-\frac{z^{2}}{z_{R}^{2}}\right)+2 g \frac{z y}{z_{R}}\right] \sin \varphi_{-}\right\}, \\
H_{z}=\frac{H_{x}=-E_{y},}{\pi w^{2}(z)}\left\{\left[-g x\left(1-\frac{z^{2}}{z_{R}^{2}}\right)-2 \frac{z y}{z_{R}}\right] \cos \varphi_{-} .\right. \\
\left.+\left[y\left(1-\frac{z^{2}}{z_{R}^{2}}\right)-2 g \frac{z x}{z_{R}}\right] \sin \varphi_{-}\right\},
\end{gathered}
$$

where

$$
\tilde{E}_{0}(\mathbf{r}, t)=E_{0} \frac{w_{0}^{2}}{w^{2}(z)} e^{-\frac{\rho^{2}}{w^{2}(z)}} f(\varphi)
$$

is the envelope function, $g$ is the parameter of ellipticity; $g=0$ corresponds to a linear polarization and $g=1-$ circular polarization, $E_{0}$ is the electric field amplitude, $\rho^{2}=x^{2}+y^{2}, w(z)=w_{0} \sqrt{1+z^{2} / z_{R}^{2}}$, where $z_{R}=\pi w_{0}^{2} / \lambda$ is the Rayleigh length of the focused laser pulse with the waist $w_{0}$ in the focal plane $z=0$, and $\varphi_{-}=\varphi-$ $k w_{0}^{2} \rho^{2} /\left[2 z w^{2}(z)\right]$ is the deformed phase. The laser pulse has temporal profile $f(\varphi)=\cosh ^{-1}(\varphi / \delta)$ with the pulse width $\delta=4$.

The carbon target is located at $z_{t}=-150 \lambda$. For the numerical calculations the total number of macroparticles is taken to be $4 \times 10^{4}$ and uniformly distributed in the target. The set of equations for the macroparticles has been solved using a standard fourth-order Runge-Kutta algorithm.

As the employed laser pulse is of nonplane configuration, particle acceleration will be experienced even in the field of a single pulse. However, the latter is small in agreement with the above mentioned Lawson-Woodward theorem [8]. This has been explicitly checked performing simulations with a single pulse given by Eqs. (2)-(6). For this setup the energy distribution in the accelerated electron layer is shown in Fig. 2 for various $z$. Calculation have been made for the circularly polarized wave with $w_{0}=40 \lambda$ and $\xi=20\left(I_{\max } \simeq 1.7 \times 10^{21} \mathrm{~W} \mathrm{~cm}^{-2}\right)$. As Fig. 2 evidences, 


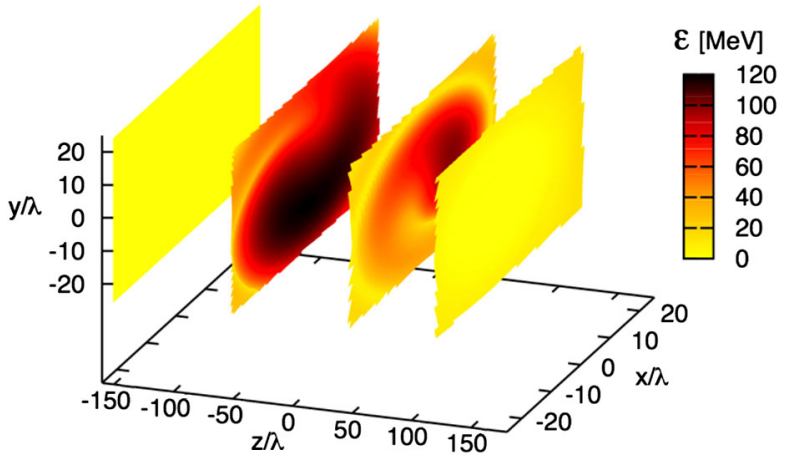

FIG. 2. Energy distribution in accelerated electron layer is shown at various $z$ for circularly polarized wave with $w_{0}=40 \lambda$ and $\xi=20$. There is no reflector-target.

the residual acceleration due to nonplanarity of the focused laser beam is small. Meanwhile, as is seen from this figure in the laser field electrons reach ultrarelativistic energies. Thus, to keep the energy gained from the wave field we propose to place a high- $\mathcal{Z}$ target near the position $z=z_{m}$ where electrons gain maximal amount of energy from the laser pulse. The purpose of the second target is twofold. First, it acts as a reflector for laser pulse, being practically transparent for electron bunch. As a consequence, we will get field-free ultrarelativistic electron bunch in the high- $\mathcal{Z}$ target. Second, within this target with sufficiently large thickness the generation of $\gamma$-ray and positrons take place due to the electron bunch-high- $\mathcal{Z}$ ion collisions. Due to high density and thickness (which should be much larger than skin depth) of second target we will consider it as a perfect reflector. Hence, one should solve the equations (1) taking into account also reflected wave. For the total electric $\mathbf{E}^{\text {(tot) }}$ and magnetic $\mathbf{H}^{\text {(tot) }}$ fields we will have: for $z>z_{m}$

$$
\mathbf{E}^{(\mathrm{tot})}=0, \quad \mathbf{H}^{(\mathrm{tot})}=0
$$

while for $z \leq z_{m}$

$E_{x, y}^{(\mathrm{tot})}(\mathbf{r}, t)=E_{x, y}(x, y, z, t)-E_{x, y}\left(x, y,-z+2 z_{m}, t\right)$,

$E_{z}^{(\mathrm{tot})}(\mathbf{r}, t)=E_{z}(x, y, z, t)+E_{z}\left(x, y,-z+2 z_{m}, t\right)$,

$H_{x, y}^{\text {(tot) }}(\mathbf{r}, t)=H_{x, y}(x, y, z, t)+H_{x, y}\left(x, y,-z+2 z_{m}, t\right)$,

$H_{z}^{(\mathrm{tot})}(\mathbf{r}, t)=H_{z}(x, y, z, t)-H_{z}\left(x, y,-z+2 z_{m}, t\right)$.

The typical picture of acceleration dynamics is shown in Fig. 3, where energy distribution in accelerated electron layer is shown for various $z$ for the circularly polarized wave with $w_{0}=40 \lambda$. The reflector-target is located at $z_{m}=0$. As is seen from Fig. 3, after the passing the second target we have field-free ultrarelativistic electron bunch.

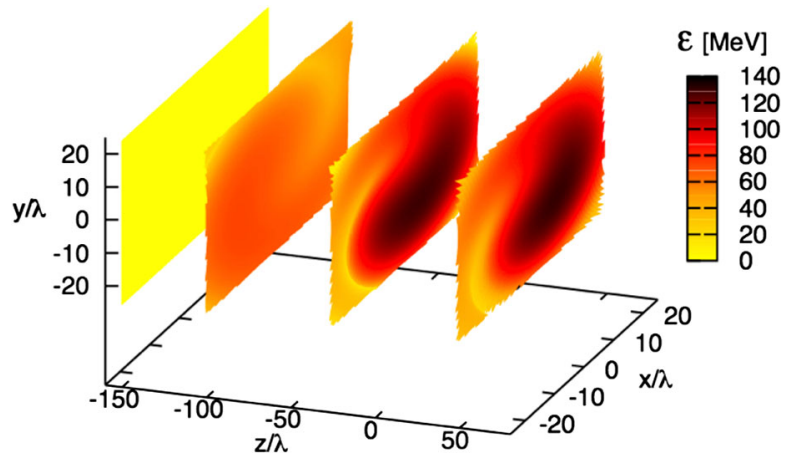

FIG. 3. Energy distribution in accelerated electron layer is shown at various $z$ for circularly polarized wave with $w_{0}=40 \lambda$ and $\xi=20$. Reflector-target is located at $z_{m}=0$.

With the reduction of laser beam waist $w_{0}$ the distance $\left|z_{t}-z_{m}\right|$, where the electron bunch gains maximum energy, decreases. In Fig. 4, we show the results for circularly polarized wave with $w_{0}=15 \lambda$ and $\xi=20$. For this setup the reflector-target is located at $z_{m}=-50 \lambda$.

We have made calculations also for the linearly polarized laser beam $(g=0)$. The energy distribution in accelerated electron layer at various $z$ is shown in Fig. 5 for linearly polarized wave with $w_{0}=10 \lambda$ and

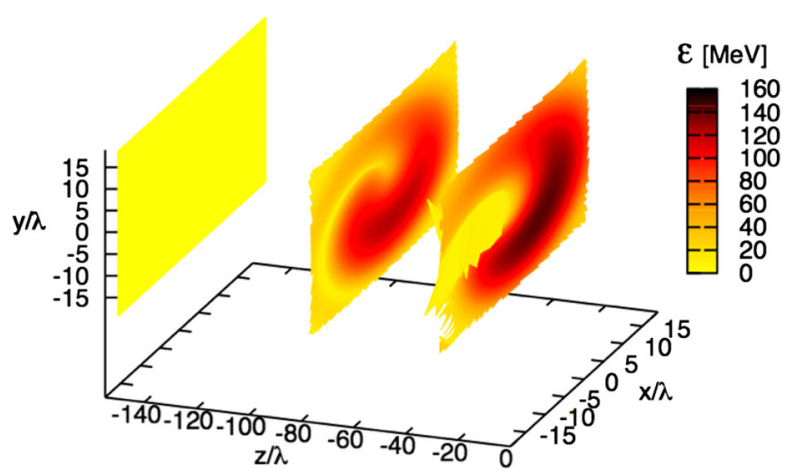

FIG. 4. Energy distribution in accelerated electron layer at various $z$ for circularly polarized wave with $w_{0}=15 \lambda$ and $\xi=20$. Reflector-target is located at $z_{m}=-50 \lambda$.

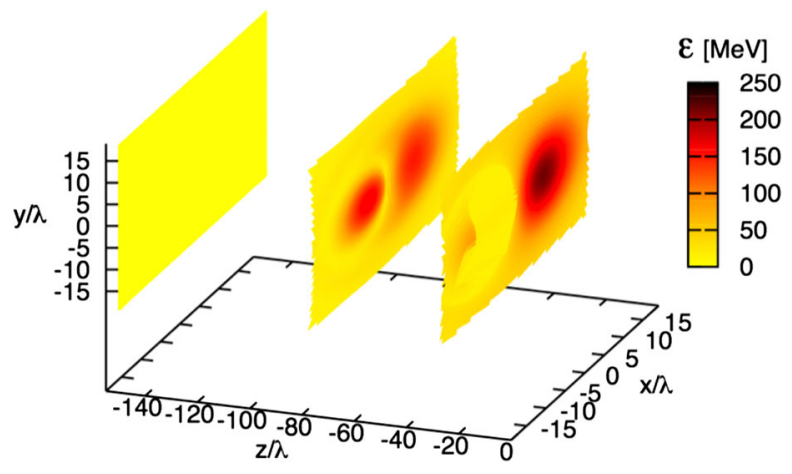

FIG. 5. Energy distribution in accelerated electron layer at various $z$ for linearly polarized wave with $w_{0}=10 \lambda$ and $\xi=30$. 

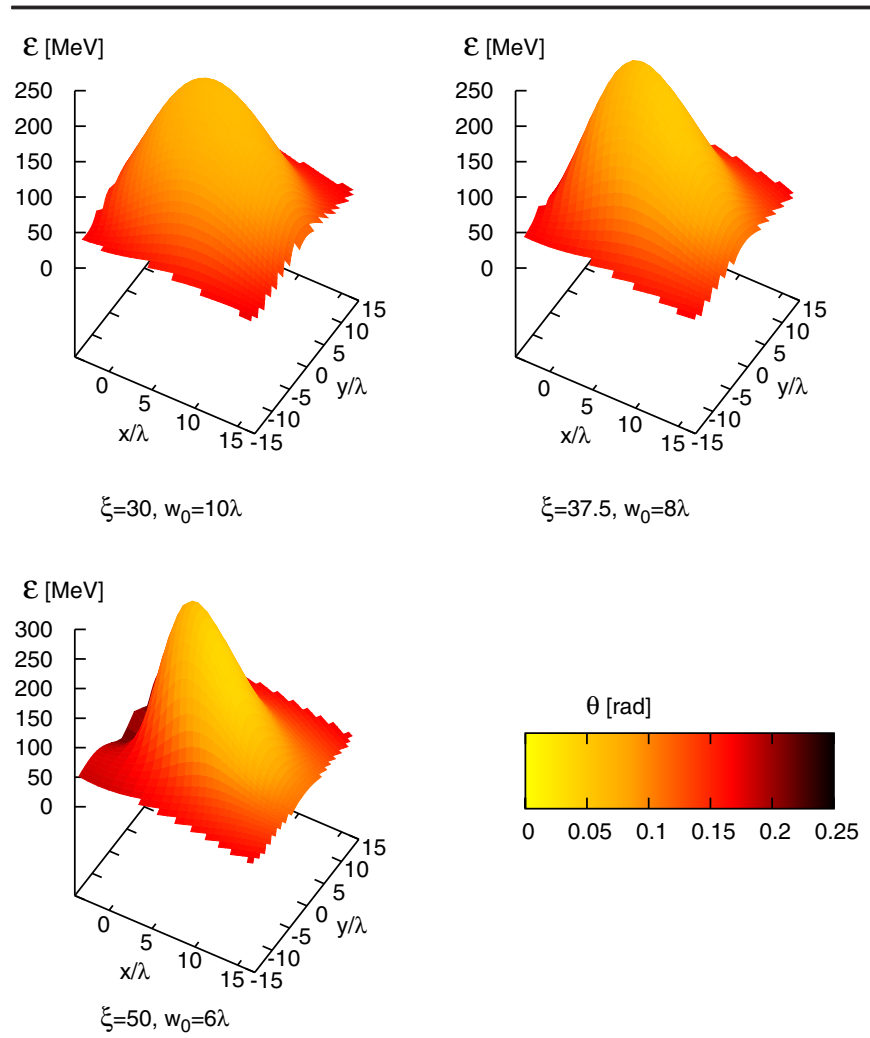

FIG. 6. The energy and angular distribution in accelerated electron layer near the reflector target $z=-49 \lambda$ versus transversal coordinates for various laser intensities but for the same laser pulse energy. The reflector-target is located at $z_{m}=-50 \lambda$.

$\xi=30\left(I_{\max } \simeq 1.9 \times 10^{21} \mathrm{~W} \mathrm{~cm}^{-2}\right)$. The reflector-target is located at $z_{m}=-50 \lambda$. To show the dependence of generated electron bunch characteristics upon laser beam parameters, in Fig. 6 we display the energy-angular $\left[\theta=\arccos \left(p_{z} / p\right)\right]$ distributions of accelerated electrons versus the transversal position for the fixed laser pulse energy at various waists and intensities near the reflector target. As is seen from this figure, in the field-free region we have an ultrarelativistic electron bunch of energies $\sim 200-300 \mathrm{MeV}$ and angular divergence $\delta \theta \sim 0.1 \mathrm{rad}$. The bunch transversal size is about $2 w_{0}$. Thus, with the focusing of a laser beam one can increase the peak energy and reduce the transversal emittance of the produced electron bunch.

Note that in the high- $Z$ target due to the electron bunchion collisions the electrons will lose energy because of generation of $\gamma$-quanta and positrons. Hence, if one expects to obtain unperturbed electron bunch, the target thickness $L_{t}$ should be much smaller than the radiation length $\mathcal{L}_{\text {rad }}$. The reflector target should be thicker than the skin depth. For the high- $Z$ targets $(Z \sim 70)$ a thickness $L_{t} \approx \lambda / 20=$ $40 \mathrm{~nm} \sim 10^{-5} \mathcal{L}_{\text {rad }}$ is sufficient to fully reflect the drive pulse. On the other hand, the relativistic electron bunch will passe such reflector target unperturbed. For the targets with $L_{t} \lesssim \mathcal{L}_{\text {rad }}$ along with primary bunch one will have substantial amounts of produced high energy photons and positrons. This scenario is also of interest and will be considered in the next section.

\section{PRODUCTION OF HARD $\gamma$-QUANTA AND POSITRONS WITH LASER GENERATED ULTRARELATIVISTIC ELECTRON BUNCHES}

As is seen from Figs. 2-5, the energy distribution of accelerated electrons obtained by the laser-target interaction is usually broad with almost $100 \%$ energy spread up to a cutoff energy. Such electron bunches are not suitable for coherent effects, for example, to generate coherent radiation-free electron laser. However, such bunches of high energy-density can be used to obtain high-flux of positrons, hard $\gamma$-quanta with possible applications in material science, medicine, and nuclear physics. Note that in our scheme this can be implemented by all-optical means without use of large scale facilities such as nuclear reactors or conventional particle accelerators. One can use the second target as a bremsstrahlung converter, leading to the emission of bremsstrahlung photons, the energy of which takes a maximum value equal to that of the accelerated electrons. Then, the bremsstrahlung photons interacting with ions can produce electron-positron pairs. In other words, QED cascade can be developed. Accurate calculations of $\gamma$-quanta and positrons spectra in such targets require using Monte Carlo computer codes (see, e.g., Ref. [44] and references therein). However, one can use relatively simple formulas [45] for thin targets, when target thickness is smaller than the radiation length $\mathcal{L}_{\text {rad }}$ :

$$
\mathcal{L}_{\text {rad }}=\left(4 \alpha N_{i} \mathcal{Z}^{2} r_{0}^{2} \log \frac{183}{\mathcal{Z}^{1 / 3}}\right)^{-1},
$$

where $\alpha=e^{2} / \hbar c=1 / 137$ is the fine structure constant, $N_{i}$ is the density of ions, $r_{0}$-is the electron classical radius. In particular, for gold $\left(\mathcal{Z}=79, N_{i}=5.9 \times 10^{22} \mathrm{~cm}^{-3}\right)$ $\mathcal{L}_{\text {rad }} \simeq 0.31 \mathrm{~cm}$, and for tantalum $\left(\mathcal{Z}=73, \quad N_{i}=\right.$ $5.55 \times 10^{22} \mathrm{~cm}^{-3}$ ) $\quad \mathcal{L}_{\text {rad }} \simeq 0.39 \mathrm{~cm}$. Thus, the $\gamma$-quanta number distribution $N_{\gamma}$ over energy $\varepsilon_{\gamma}$ obeys integrodifferential equation [45]:

$\frac{\partial N_{\gamma}\left(\varepsilon_{\gamma}, \zeta\right)}{\partial \zeta}=\int_{0}^{1} N_{e}\left(\frac{\varepsilon_{\gamma}}{v}, \zeta\right) \varphi_{0}(v) \frac{d v}{v}-\sigma_{0} N_{\gamma}\left(\varepsilon_{\gamma}, \zeta\right)$,

Here $\sigma_{0} \simeq 7 / 9$, and $\varphi_{0}(v)$ describes bremsstrahlung process, which in the case of complete screening is given by the formula:

$$
\varphi_{0}(v)=\frac{1}{v}\left(1+(1-v)^{2}-\varkappa_{0}(1-v)\right),
$$

where $\varkappa_{0} \simeq 0.64$. The quantity $\zeta$ in Eq. (14) is the target thickness measured in radiation lengths, $N_{e}(\mathcal{E}, \zeta)$ is the electron/positron distribution function, that should be defined self-consistently. However, for small $\zeta$, in 


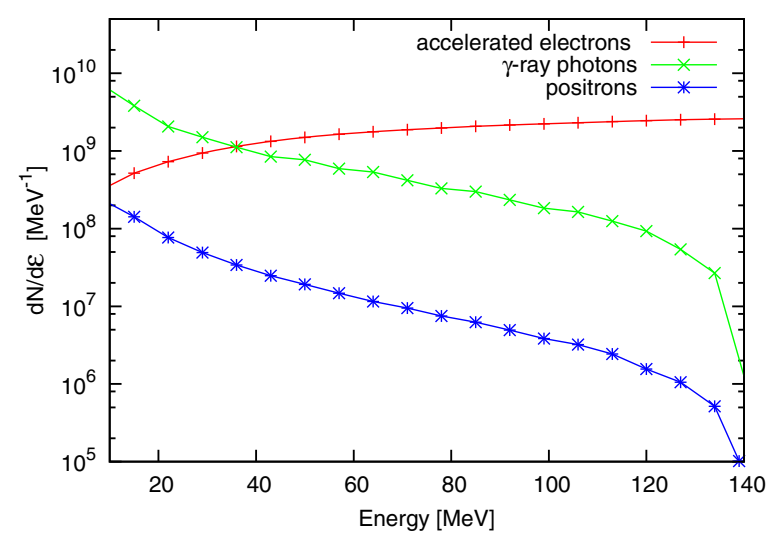

FIG. 7. In the logarithmic scale it is shown the energy distribution functions for primary accelerated electrons, produced $\gamma$-quanta and positrons for the setup of Fig. 4.

Eq. (14) we take $N_{e}(\mathcal{E}, \zeta)=N_{e}(\mathcal{E}, 0)$, where $N_{e}(\mathcal{E}, 0)$ is the distribution function at the entrance to high- $\mathcal{Z}$ target. Thus, for small $\zeta$ we have

$$
N_{\gamma}\left(\varepsilon_{\gamma}, \zeta\right) \simeq \zeta \int_{0}^{1} N_{e}\left(\frac{\varepsilon_{\gamma}}{v}, 0\right) \varphi_{0}(v) \frac{d v}{v}
$$

In the limit of low annihilation rates the positrons distribution function can be defined as

$$
\frac{\partial N_{e^{+}}(\mathcal{E}, \zeta)}{\partial \zeta}=\int_{0}^{1} N_{\gamma}\left(\frac{\mathcal{E}}{u}, \zeta\right) \psi_{0}(u) \frac{d u}{u},
$$

where $\psi_{0}(u)$ describe pair production process, which in the case of complete screening is given by the formula [45]:

$$
\psi_{0}(u)=u^{2}+(1-u)^{2}+\varkappa_{0} u(1-u)
$$

From the electron bunch distribution $N_{e}(\mathcal{E}, 0)$ and Eqs. (16) and (17), it is possible to evaluate the $\gamma$-ray and positron energy spectrum. For the target thickness we take $\zeta=0.2$. We have made calculations for the setup of Figs. 4 and 5. To obtain electron bunch distribution $N_{e}(\mathcal{E}, 0)$ at the entrance to bremsstrahlung target we considered the effective area limited to the central zone between $-20 \lambda<x, y<20 \lambda$. The results of calculations are presented in Figs. 7 and 8. In these figures, in the logarithmic scale it is shown the energy distribution functions for primary accelerated electrons, produced $\gamma$ quanta, and positrons. As is seen from these figures, through two-target scheme one can produce dense electron/positron bunches and substantial amounts of $\gamma$-quanta of energies up to $200 \mathrm{Mev}$. In particular, for Fig. 8 the number of photons in the range of $50-200 \mathrm{Mev}$ is $\sim 10^{9}$.

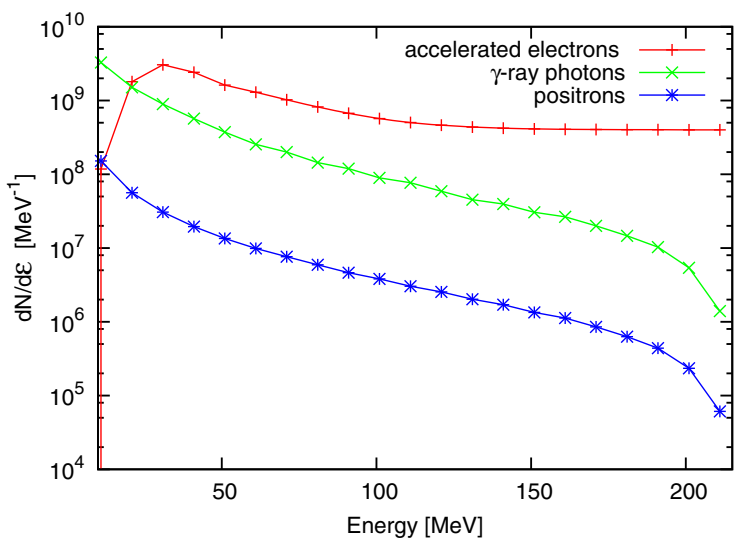

FIG. 8. Same as Fig. 7, but for the setup of Fig. 5.

\section{CONCLUSION}

We have proposed a mechanism for generation of high energy-density particles bunches from nanotargets by single laser pulse of ultrarelativistic intensities. We consider a two target scheme where one ultrathin target serves as a source of dense electron bunch. The purpose of the second target is twofold. First, it acts as a reflector for laser pulse, thus abruptly terminating the wave field and therefore allows electrons to maintain the gained energy. Second, within this target with sufficiently large thickness the intense generation of hard $\gamma$-quanta and positrons occurs due to the electron-ion collisions. In particular, the considered setup provides generation of high energy-density positrons and $\gamma$-quanta that may have applications in material science, medicine, and nuclear physics.

\section{ACKNOWLEDGMENTS}

This work was supported by the Republic of Armenia (RA) Ministry of Education and Science (MES), State Committee of Science, in the frames of the research project No. 13-1C066.

[1] V. Yanovsky et al., Opt. Express 16, 2109 (2008).

[2] J. H. Sung, S. K. Lee, T. J. Yu, T. M. Jeong, and J. Lee, Opt. Lett. 35, 3021 (2010).

[3] A. V. Korzhimanov, A. A. Gonoskov, E. A. Khazanov, and A. M. Sergeev, Phys. Usp. 54, 9 (2011).

[4] Z. Wang, C. Liu, Z. Shen, Q. Zhang, H. Teng, and Z. Wei, Opt. Lett. 36, 3194 (2011).

[5] The Extreme Light Infrastructure (ELI) project: http:// www.extreme-light-infrastructure.eu/eli-home.php.

[6] Exawatt Center for Extreme Light Studies: http://www .xcels.iapras.ru.

[7] High Power laser Energy Research: http://www.hiper-laser .org.

[8] P. M. Woodward, J. Inst. Elect. Eng. 93, 1554 (1946); J. D. Lawson, IEEE Trans. Nucl. Sci. 26, 4217 (1979). 
[9] H. K. Avetissian, Relativistic Nonlinear Electrodynamics (Springer, New York, 2006).

[10] H. K. Avetissian, S. S. Israelyan, and Kh. V. Sedrakian, Phys. Rev. ST Accel. Beams 10, 071301 (2007); H. K. Avetissian and Kh. V. Sedrakian, Phys. Rev. ST Accel. Beams 13, 101304 (2010); 13, 081301 (2010).

[11] E. Esarey, C. B. Schroeder, and W. P. Leemans, Rev. Mod. Phys. 81, 1229 (2009).

[12] E. Esarey, P. Sprangle, and J. Krall, Phys. Rev. E 52, 5443 (1995).

[13] F. V. Hartemann, S. N. Fochs, G. P. Le Sage, N. C. Luhmann, J. G. Woodworth, M. D. Perry, Y. J. Chen, and A. K. Kerman, Phys. Rev. E 51, 4833 (1995).

[14] Y. C. Huang, D. Zheng, W. M. Tulloch, and R. L. Byer, Appl. Phys. Lett. 68, 753 (1996).

[15] G. Malka, E. Lefebvre, and J. L. Miquel, Phys. Rev. Lett. 78, 3314 (1997).

[16] B. Quesnel and P. Mora, Phys. Rev. E 58, 3719 (1998).

[17] G. V. Stupakov and M. S. Zolotorev, Phys. Rev. Lett. 86, 5274 (2001).

[18] W. D. Kimura et al., Phys. Rev. Lett. 86, 4041 (2001).

[19] P. X. Wang, Y. K. Ho, X. Q. Yuan, Q. Kong, N. Cao, A. M. Sessler, E. Esarey, and Y. Nishida, Appl. Phys. Lett. 78, 2253 (2001).

[20] Y. I. Salamin and C. H. Keitel, Phys. Rev. Lett. 88, 095005 (2002).

[21] S. X. Hu and A. F. Starace, Phys. Rev. Lett. 88, 245003 (2002).

[22] T. Plettner, R. L. Byer, E. Colby, B. Cowan, C. M. S. Sears, J. E. Spencer, and R. H. Siemann, Phys. Rev. Lett. 95, 134801 (2005).

[23] C. Varin, M. Piché, and M. A. Porras, Phys. Rev. E 71, 026603 (2005).

[24] Y. I. Salamin, Phys. Rev. A 73, 043402 (2006); Y. I. Salamin, Opt. Lett. 32, 90 (2007).

[25] A. Karmakar and A. Pukhov, Laser Part. Beams 25, 371 (2007).

[26] P.-L. Fortin, M. Piché, and C. Varin, J. Phys. B 43, 025401 (2010).
[27] L. J. Wong and F. X. Kärtner, Opt. Express 18, 25035 (2010).

[28] F. Terranova, Phys. Rev. ST Accel. Beams 17, 071301 (2014).

[29] J. A. Edinghofer and R. H. Pantell, J. Appl. Phys. 50, 6120 (1979).

[30] T. Plettner, R. L. Byer, E. Colby, B. Cowan, C. M. S. Sears, J. E. Spencer, and R. H. Siemann, Phys. Rev. Lett. 95, 134801 (2005).

[31] Yu. M. Mikhailova, V. T. Platonenko, and S. G. Rykovanov, JETP Lett. 81, 571 (2005).

[32] A. S. Pirozhkov, S. V. Bulanov, T. Zh. Esirkepov, M. Mori, A. Sagisaka, and H. Daido, Phys. Plasmas 13, 013107 (2006).

[33] S. G. Rykovanov, J. Schreiber, and J. Meyer-ter-Vehn, New J. Phys. 10, 113005 (2008).

[34] H.-C. Wu, J. Meyer-ter-Vehn, J. Fernández, and B. M. Hegelich, Phys. Rev. Lett. 104, 234801 (2010).

[35] D. Kiefer et al., Eur. Phys. J. D 55, 427 (2009).

[36] V. V. Kulagin, V. A. Cherepenin, M. S. Hur, and H. Suk, Phys. Rev. Lett. 99, 124801 (2007).

[37] V. V. Kulagin, V. A. Cherepenin, Y. V. Gulyaev, V. N. Kornienko, K. H. Pae, V. V. Valuev, J. Lee, and H. Suk, Phys. Rev. E 80, 016404 (2009).

[38] S. Humphries, Charged Particle Beams (Wiley, New York, 1990).

[39] J. Koga, T. Zh. Esirkepov, and S. V. Bulanov, Phys. Plasmas 12, 093106 (2005).

[40] J. P. Verboncoeur, A. B. Langdon, and N. T. Gladd, Comput. Phys. Commun. 87, 199 (1995).

[41] B. Rau, T. Tajima, and H. Hojo, Phys. Rev. Lett. 78, 3310 (1997).

[42] A. Macchi, F. Cattani, T. V. Liseykina, and F. Cornolti, Phys. Rev. Lett. 94, 165003 (2005).

[43] K. T. McDonald, arXiv:physics/0003056v2.

[44] S. Agostinelli et al., Nucl. Instrum. Methods Phys. Res., Sect. A 506, 250 (2003).

[45] B. Rossi and K. Greisen, Rev. Mod. Phys. 13, 240 (1941). 